"TRIFLE' CENTRE

FOR APPLIED MATHEMATICS IN NATURAL SCIENCE

On infinitesimal increase of volumes of morphological transforms

Markus Kiderlen and Jan Rataj

Research Report

No. 12 | November 2005 


\section{On infinitesimal increase of volumes of morphological transforms}

This Thiele Research Report is also Research Report number 465 in the Stochastics Series at Department of Mathematical Sciences, University of Aarhus, Denmark. 



\title{
ON INFINITESIMAL INCREASE OF VOLUMES OF MORPHOLOGICAL TRANSFORMS
}

\author{
MARKUS KIDERLEN AND JAN RATAJ
}

\begin{abstract}
Let $B$ ("black") and $W$ ("white") be disjoint compact test sets in $\mathbb{R}^{d}$ and consider the volume of all its simultaneous shifts keeping $B$ inside and $W$ outside a compact set $A \subset \mathbb{R}^{d}$. If the union $B \cup W$ is rescaled by a factor tending to zero, then the rescaled volume converges to a value determined by the surface area measure of $A$ and the support functions of $B$ and $W$, provided that $A$ is regular enough (e.g. polyconvex). An analogous formula is obtained for the case when the conditions $B \subset A$ and $W \subset A^{C}$ are replaced with prescribed threshold volumes of $B$ in $A$ and $W$ in $A^{C}$.

Applications in stochastic geometry are discussed. Firstly, the hit distribution function of a random set with an arbitrary compact structuring element $B$ is considered. Its derivative at 0 is expressed in terms of the rose of directions and $B$. An analogue result holds for the hit-or-miss function. Secondly, in a desing based setting, different random digitizations of a deterministic set $A$ are treated. It is shown how the number of configurations in such a digitization is related to the surface area measure of $A$ as the lattice distance converges to zero.
\end{abstract}

\section{Dedicated to Rolf Schneider on the occasion of his 65th birthday}

\section{INTRODUCTION}

The idea to approximate the surface area of a set $A \subset \mathbb{R}^{d}$ by the volume increase of its dilation with an infinitesimal ball,

$$
\lim _{\varepsilon \rightarrow 0_{+}} \frac{\mathcal{H}^{d}\left(A \oplus \varepsilon B^{d}\right)-\mathcal{H}^{d}(A)}{\varepsilon}
$$

( $B^{d}$ stands for the Euclidean unit ball here) goes back to Minkowski. When the unit ball $B^{d}$ is replaced by a unit line segment of direction $u \in \mathbb{S}^{d-1}$, (1) equals the intersection density of $\partial A$ with lines of direction $u$, which is representable as the integral

$$
\int_{\mathbb{S}^{d-1}}|u \cdot n| S_{d-1}(A ; d n)
$$

with respect to the surface area measure $S_{d-1}(A ; \cdot)$ of $A$ for certain classes of sets with regular boundaries. This is used in stereology for the estimation of the surface area measure. It is, however, well known that line intersection densities can give us information only about the symmetrization $S_{d-1}(A ; \cdot)+S_{d-1}(-A ; \cdot)$, and not about $S_{d-1}(A ; \cdot)$ itself. Schneider [19] proposed a modified estimator involving the outer normal direction of $A$ at the intersection points with the test lines. Replacing $B^{d}$ in

Key words and phrases. Surface area measure, dilation, erosion, hit-or-miss transform, volumethreshold, digitization, contact distribution, compact structuring element, rose of direction.

The first author was supported by the Carlsberg foundation and the second author was supported partially by grants MSM 0021620839 of the Czech Ministery of Education and GAČR 201/03/0946 of the Grant Agency of Czech Republic. 
(1) by a general (centered) convex body B, Rataj [14, Corollary 4.2] showed

$$
\lim _{\varepsilon \rightarrow 0_{+}} \frac{\mathcal{H}^{d}(A \oplus \varepsilon B)-\mathcal{H}^{d}(A)}{\varepsilon}=2 \int_{S^{d-1}} h(B, n) S_{d-1}(A ; d n),
$$

for a class of $\mathcal{U}_{P R}$ sets $A$, where $h(B, n)$ is the support function of $B$; see also Hug [5] for a similar result for $A$ from the convex ring. It is not required here that $B$ is symmetric, as the centering condition only means that the Steiner point of $B$ coincides with the origin $o$. By a result of Schneider [18, p. 283], the integrals in (2) determine $S_{d-1}(A, \cdot)$ uniquely, if known for all centered convex bodies $B$. Hence this result can be used to establish estimators for $S_{d-1}(A, \cdot)$ from observations of $\mathcal{H}^{d}(A \oplus \varepsilon B)-\mathcal{H}^{d}(A)=\mathcal{H}^{d}([A \oplus \varepsilon B] \backslash A)$ for small $\varepsilon$ and several test sets $B$. Corollary 2.(2) shows that $(2)$ holds for arbitrary compact sets $B \neq \emptyset$, where $h(B, \cdot)$ is the support function of the convex hull of $B$. Motivated by mathematical morphology, we replace the set $(A \oplus \varepsilon B) \backslash A$ by the hit-or-miss transform of $A$,

$$
[A \ominus \varepsilon B] \backslash[A \oplus \varepsilon W] .
$$

Here

$$
A \ominus B=\left\{x \in \mathbb{R}^{d}: x+\check{B} \subset A\right\}
$$

is the dilation of $A$ with $\check{B}$, i.e. the set of all translation vectors such that the translation of $\check{B}=\{-b: b \in B\}$ is completely contained in $A$. The hit-or-miss transform depends on $A$ and on a pair of compact structuring elements $(B, W)$. Kiderlen and Jensen [10] showed the asymptotic formula

$$
\lim _{\varepsilon \rightarrow 0_{+}} \frac{1}{\varepsilon} \mathcal{H}^{d}([A \ominus \varepsilon B] \backslash[A \oplus \varepsilon W])=\int_{\mathbb{S}^{d-1}}(-h(\check{B} \oplus W, n))^{+} S_{d-1}(A, d n)
$$

in the planar case $(d=2)$ for finite sets $B$ and $W$. Here $a^{+}=\max \{0, a\}$ is the positive part of $a \in \mathbb{R}$. This result, which was originally formulated in terms of random sets, holds in arbitrary dimensions and for all nonempty compact subsets $B, W$ of $\mathbb{R}^{d}$; see Corollary 2.(3), below. We show in Proposition 5 , that $S_{d-1}(A, \cdot)$ is determined, if the left hand side of (3) is known for all finite sets $B$ and $W$. It is actually enough to take only those pairs $(B, W)$ into account, for which $\check{B} \oplus W$ has at most three elements. The asymptotic results (2) and (3) are special cases of Theorem 1, where local versions and more involved combinations of erosions and dilations are studied.

Given $0<\theta \leq 1$ and a Borel-measurable set $Q \subset \mathbb{R}^{d}$ of positive volume, Molchanov [13] defines the set

$$
A \oslash_{\theta} Q=\left\{x \in \mathbb{R}^{d}: \mathcal{H}^{d}(A \cap(x+\check{Q})) \geq \theta \mathcal{H}^{d}(Q)\right\},
$$

which is the set of all "locations" $x$ such that the volume fraction of $A$-points in $x+\check{Q}$ is at least $\theta$. We call $A \oslash_{\theta} Q$ the volume-threshold set of $A$ (with threshold parameter $\theta$ and sampling element $Q$ ). We have

$$
A \ominus Q \subseteq A \oslash_{1} Q \subseteq A \oslash_{\theta} Q \subseteq A \oplus Q
$$

If $A$ is topologically regular, then $A \oslash_{1} Q=A \ominus Q$ for closed $Q$. This shows that volume-thresholding defines a family of operations "between" erosion and dilation. In Theorem 2 and its corollaries, we extend Theorem 1 to certain combinations of dilations, erosions and volume-threshold sets. 
The last two sections are devoted to applications in random set theory and digital stereology. In Section 5, a (not necessarily stationary) random closed set $Z$ is considered. The hit distribution function with structuring element $B$ at $z \in \mathbb{R}^{d}$ can be defined by

$$
H_{B}(z, t)=P(Z \cap(z+t B) \neq \emptyset \mid z \notin Z) .
$$

We assume here $o \in B$. For star shaped $B$, this function coincides with the contact distribution function, which is an important summary statistics and has been studied extensively; see e.g. Hug \& Last [6], Hug et al. [7], [9], Stoyan et al. [21]. For the Boolean model, Molchanov [12] gives a survey of possible applications of this function and Hug et al. [8] consider generalized contact distribution functions. Theorem 3 shows that under weak conditions, (2) transfers to random sets, where the left hand side now involves the derivative of $H_{B}(z, \cdot)$ at $t=0$ and the right hand side depends on the (position dependent) rose of directions of $Z$. Similarly, Theorem 4 is the random set counterpart of (3), expressing the derivative at $t=0$ of the hit-or-miss function

$$
G_{(B, W)}(z, t)=P\left(z+t B \subseteq Z, z+t W \subseteq \mathbb{R}^{d} \backslash Z \mid z \notin Z\right)
$$

in terms of an integral with respect to the rose of directions. Section 6 exemplifies applications in design based digital stereology, where the set $A$ of interest is assumed to be deterministic and compact. In digital stereology, we assume that the set $A$ is not observable directly, but instead, only a digitization of $A$ is available. In the simplest case, the digitization of $A$ is given by all points of a uniformly translated random lattice in $\mathbb{R}^{d}$ that are contained in $A$ : If $\mathbb{Z}^{d}$ is the standard lattice and $\xi$ is a uniform random vector in the unit cube $[0,1]^{d}$, we assume that the (random) hitor-miss digitization $A \cap t\left(\xi+\mathbb{Z}^{d}\right)$ is observable, where $t>0$ is a scaling parameter. Now if $B, W \subset \mathbb{Z}^{d}$ are non-empty and $x \in t\left(\xi+\mathbb{Z}^{d}\right)$, then the indicator function

$$
\mathbf{1}_{\left\{x+t B \subseteq A, x+t W \subseteq \mathbb{R}^{d} \backslash A\right\}}
$$

can be determined from the digitization $A \cap t\left(\xi+\mathbb{Z}^{d}\right)$. The number $N_{t}$ of all $x \in t(\xi+$ $\mathbb{Z}^{d}$ ) for which this indicator function is 1 can be seen as the number of occurrences of point configurations which coincide up to lattice translations with $(t B, t W)$. As the expected value of $t^{d} N_{t}$ is $\mathcal{H}^{d}([A \ominus t \check{B}] \backslash[A \oplus t \check{W}])$, (3) implies that $t^{d-1} N_{t}$ is an asymptotically unbiased estimator for

$$
\int_{\mathbb{S}^{d-1}}(-h(B \oplus \check{W}, n))^{+} S_{d-1}(A, d n),
$$

as the lattice distance $t$ converges to 0 . Theorem 5 states this result in a more general form, where the standard lattice $\mathbb{Z}^{d}$ is replaced by a general regular lattice $\mathbb{L}$ and different digitization schemes are used. One of them is the so-called volume-threshold digitization, which deserves particular emphasis, due to its practical importance: In material sciences, computer tomography is an increasingly important tool to obtain a digitization of a three-dimensional structure $A$ in a non-invasive way. Standard methods yield a gray-tone digitization of $A$, where the gray-value in a lattice point $p \in t \mathbb{Z}^{3}$ corresponds (approximately) to the volume fraction of $A$ in a voxel $p+t Q$ centered at $p$. In most cases, $Q$ is just an axis parallel unit cube with midpoint at the origin. To obtain a 0-1-image, this gray-tone digitization is then usually thresholded with a threshold parameter $\theta$ between 0 and 1 . Mathematically speaking, instead of observing the hit-or-miss digitization, we observe the set $\left[A \oslash_{\theta} t Q\right] \cap t \mathbb{Z}^{d}$. This 
explains, why our method yields asymptotic results for data obtained from tomography. Hall \& Molchanov [4] derived a result on volume estimation from $\left[A \oslash_{\theta} t Q\right] \cap t \mathbb{Z}^{d}$, which we extend to a more general set class in Proposition 6.

At the beginning of this introduction, we mentioned the condition that $A$ is supposed to belong to a class of sets with sufficiently smooth boundary. As a rule, weaker conditions on $B$ require stronger smoothness conditions on $A$ for the above results to hold. For example, it follows from Hug et al. [9, equation (4.4)] that (2) with $B=B^{d}$ holds for a very large class of compact sets $A$. (To be precise it holds for all compact sets $A$ whose support measures all are finite signed measures; thus excluding in particular certain fractal sets.) If $B$ is assumed to be compact and convex (with $O$ in its interior), then (2) is known to hold for $A$ in the convex ring; see [6]. For general compact $B$, we will assume that $A$ belongs to a class, which is for brevity named the class of "gentle" sets, cf. Section 2; it encompasses topologically regular sets from the convex ring (Proposition 2) and certain unions of sets with positive reach (Propositions 1 and 3). A key tool in our investigations is a general version of the Steiner formula in Hug et al. [9]. We recall a special case of this result in Proposition 4, where it is applied to the boundary $\partial A$ of the gentle set $A$.

\section{Gentle Sets}

The setting is the Euclidean space $\mathbb{R}^{d}$ with scalar product $u \cdot v, u, v \in \mathbb{R}^{d}$. We use the notation $\mathcal{H}^{k}$ for the $k$-dimensional Hausdorff measure in $\mathbb{R}^{d}$, so that $\mathcal{H}^{d}$ is the Lebesgue measure. We say that a vector $u$ is tangent to $A \subset \mathbb{R}^{d}$ if there exists a sequence $a_{i} \rightarrow a, a_{i} \in A \backslash\{a\}$, and a sequence $r_{i}$ of positive numbers such that $r_{i}\left(a_{i}-a\right) \rightarrow u, i \rightarrow \infty$. We denote by Tan $(A, a)$ the tangent cone of $A$ at $a \in \mathbb{R}^{d}$ (this is always a closed cone). The normal cone to $A$ at $a$, Nor $(A, a)$, is the dual cone to $\operatorname{Tan}(A, a)$, i.e.,

$$
\operatorname{Nor}(A, a)=\left\{v \in \mathbb{R}^{d}: u \cdot v \leq 0 \text { for any } u \in \operatorname{Tan}(A, a)\right\} .
$$

The exoskeleton $\operatorname{exo}(A)$ of a closed set $A \subseteq \mathbb{R}^{d}$ is the set of all $z \in \mathbb{R}^{d} \backslash A$ which do not have a unique nearest point in $A$. The set $\operatorname{exo}(A)$ is measurable and its $d$-dimensional Hausdorff-measure is $\mathcal{H}^{d}(\operatorname{exo}(A))=0$. The metric projection $\xi_{A}: \mathbb{R}^{d} \backslash \operatorname{exo}(A) \rightarrow A$ is defined so that $\xi_{A}(a) \in A$ is the unique nearest point to $a$ in $A$. The reduced normal bundle of $A$ is (see [9])

$$
N(A):=\left\{\left(\xi_{A}(z), \frac{z-\xi_{A}(z)}{\left|z-\xi_{A}(z)\right|}\right): z \notin A \cup \operatorname{exo}(A)\right\} .
$$

(Note that $N(A)$ is called normal bundle in [9]; we use the adjective 'reduced' in order to avoid confusion with the unit normal bundle nor $A$, which will be defined later.) We have $N(A) \subset \partial A \times \mathbb{S}^{d-1}$, where $\mathbb{S}^{d-1}$ is the unit sphere in $\mathbb{R}^{d}$. The reach function of $A$,

$$
\delta(A ; a, n):=\inf \{t \geq 0: a+t n \in \operatorname{exo}(A)\}
$$

is positive for all $(a, n) \in N(A)$. The reach of a set $A$ (denoted reach $A$ ) is the distance between $A$ and its exoskeleton $\operatorname{exo}(A)$ (we set reach $A=\infty$ if $\operatorname{exo}(A)$ is empty, which happens if and only if $A$ is convex). If reach $A>0$ then $A$ is closed and $\operatorname{Tan}(A, a)$ is a convex cone for any $a \in A$ (see [1]).

Let $A^{*}=\overline{\mathbb{R}^{d} \backslash A}$ be the closure of the set-complement of $A$.

A set $A \subseteq \mathbb{R}^{d}$ is called topologically regular, if it is the closure of its interior and $A$ is called full-dimensional if its tangent cone $\operatorname{Tan}(A, a)$ is full dimensional (i.e., it 
spans the whole $\mathbb{R}^{d}$ ) for $\mathcal{H}^{d-1}$-almost all $a \in A$. We say that a closed set $A \subseteq \mathbb{R}^{d}$ is gentle if

(i) $\mathcal{H}^{d-1}\left(N(\partial A) \cap\left(B \times \mathbb{S}^{d-1}\right)\right)<\infty$, for all bounded Borel sets $B \subseteq \mathbb{R}^{d}$,

(ii) for $\mathcal{H}^{d-1}$-almost all $a \in \partial A$, there are non-degenerate balls $B_{i}$ and $B_{o}$ containing both $a$ with $B_{i} \subseteq A$ and int $B_{o} \subseteq \mathbb{R}^{d} \backslash A$.

If $A$ is a closed gentle set, then $A$ is full-dimensional due to (ii). $A$ need not be topologically regular (consider a singleton), but it satisfies $\mathcal{H}^{d-1}(A \backslash \overline{\operatorname{int} A})=0$. Furthermore, (ii) implies that the normalized vector $n$ pointing from $a$ to the midpoint of $B_{o}$ satisfies $(a, n) \in N(A)$, and we have $\delta(A ; a, n)>0$. As the balls $B_{i}$ and $B_{o}$ must touch in $a$, we also have $\delta\left(A^{*} ; a,-n\right)>0$ and $n$ does not depend on the special choice of $B_{i}$ and $B_{o}$. We may therefore set $n(A ; a):=n$ and use henceforth that the function $n(A ; \cdot)$ is defined $\mathcal{H}^{d-1}$-almost everywhere on $\partial A$. In particular, this implies that the positive boundary

$$
\partial^{+} A:=\left\{a \in \partial A:(a, n) \in N(A) \text { for some } n \in \mathbb{S}^{d-1}\right\}
$$

satisfies

$$
\mathcal{H}^{d-1}\left(\partial A \backslash \partial^{+} A\right)=0 .
$$

Let $C_{d-1}(A, \cdot)$ be the image measure of $\mathcal{H}^{d-1}$ on $\partial A$ under the mapping $a \mapsto$ $(a, n(A ; a))$ (this function is measurable, see $\left[9\right.$, Lemma 6.3]). $C_{d-1}(A, \cdot)$ vanishes outside $N(A)$.

The class of gentle closed sets is quite general, as we will see in the sequel. We will discuss its connection to usual set classes: The class of $\mathcal{U}_{P R}$ sets consists of all closed subsets $A$ of $\mathbb{R}^{d}$ which can be represented as locally finite unions $A=\bigcup_{i=1}^{\infty} A_{i}$ of sets with positive reach so that for any finite index set $I, \bigcap_{i \in I} A_{i}$ has positive reach whenever it is non-empty. As convex bodies (compact convex subsets of $\mathbb{R}^{d}$ ) have positive reach, sets in the extended convex ring (locally finite unions of convex bodies) are elements of $\mathcal{U}_{P R}$.

For $A \in \mathcal{U}_{P R}$, the (generalized) unit normal bundle nor $A \subset \partial A \times \mathbb{S}^{d-1}$ of $A$ was defined in $[25,16]$ as the support of the index function

$$
i_{A}(a, n):=\mathbf{1}_{A}(a)\left(1-\lim _{r \rightarrow 0_{+}} \lim _{s \rightarrow 0_{+}} \chi(A \cap B(a+((r+s) n, r))),\right.
$$

$a \in \mathbb{R}^{d}, n \in \mathbb{S}^{d-1}(B(y, t)$ denotes the closed ball of center $y$ and radius $t$ and $\chi$ stands for the Euler-Poincaré characteristic). Note that if reach $A>0$ then

$$
\text { nor } A=N(A)=\left\{(a, n): a \in \partial A, n \in \mathbb{S}^{d-1} \cap \operatorname{Nor}(A, a)\right\} \text {. }
$$

The tangent cone of nor $A$ is a $(d-1)$-dimensional subspace at $\mathcal{H}^{d-1}$-almost all points $(a, n) \in \operatorname{nor} A$ with a basis

$$
\left(\frac{1}{\sqrt{1+k_{i}(a, n)^{2}}} b_{i}(a, n), \frac{k_{i}(a, n)}{\sqrt{1+k_{i}(a, n)^{2}}} b_{i}(a, n)\right), \quad i=1, \ldots, d-1,
$$

where $k_{i}(a, n) \in(-$ reach $A, \infty]$ are the (generalized) principal curvatures and $b_{i}(a, n)$ the corresponding principal directions of $A$ at $(a, n)$ (see $[24,16])$. (We set $\frac{1}{\sqrt{1+\infty^{2}}}=0$ and $\frac{\infty}{\sqrt{1+\infty^{2}}}=1$.) 
Remarks:

- Topologically regular $\mathcal{U}_{P R^{-}}$sets need not be full-dimensional. As a counterexample consider the set $A=\left\{(x, y) \in[0,1] \times \mathbb{R}:|y| \leq \operatorname{dist}(x, C)^{2}\right\}$, where $C$ is a compact totally disconnected subset of $[0,1]$ with positive Lebesgue measure. (Indeed, if $x \notin C$ and $0<y<\operatorname{dist}(x, C)$ then $(x, y) \in \operatorname{int} A$ which shows that $A$ is topologically regular. On the other hand, if $x \in C$ then $\operatorname{Tan}(A,(x, 0))=\mathbb{R} \times\{0\}$, hence $A$ is not full-dimensional.)

- If $A$ is a full-dimensional gentle $\mathcal{U}_{P R^{-}}$set, the measure $C_{d-1}(A, \cdot)$ is just the usual curvature measure of $A$ (of order $(d-1)$ ).

Proposition 1. Any full-dimensional $\mathcal{U}_{P R}$-set $A \subseteq \mathbb{R}^{d}$ satisfies condition (ii).

Proof. Let $A=\bigcup_{i} A_{i}$ be a $\mathcal{U}_{P R}$-representation of a full-dimensional set $A \in \mathcal{U}_{P R}$. Since the normal cone $\operatorname{Nor}(C, a)$ of a set $C \subseteq \mathbb{R}^{d}$ with positive reach has dimension at most 1 at $\mathcal{H}^{d-1}$-almost all boundary points $a$ of $C$ (see $[1, \S 4.15$ (3)]), we have for $\mathcal{H}^{d-1}$-almost all $a \in \partial A$,

$$
\operatorname{dim} \operatorname{Nor}\left(\bigcap_{i \in I} A_{i}, a\right)=1
$$

for any index set $I$ with $a \in \bigcap_{i \in I} A_{i}$. Fix such a point $a \in \partial A$ and denote $I_{0}=\{i$ : $\left.a \in A_{i}\right\}$. It has been shown in the proof of [15, Theorem 1] that $A$ coincides at some neighbourhood of $a$ with the union of those sets $A_{i}, i \in I_{0}$, with full-dimensional tangent cones $\operatorname{Tan}\left(A_{i}, a\right)$. Let $n \in \mathbb{S}^{d-1}$ be the unique unit normal vector of these sets $A_{i}$ at $a$.

We have $\delta(A ; a, n)>0$ for $\mathcal{H}^{d-1}$-almost all $a \in \partial A$ by [15, Theorem 1$]$, hence the ball $B_{o}=B(a+\delta(A ; a, n) n, \delta(A ; a, n))$ has the desired property int $B_{o} \subseteq \mathbb{R}^{d} \backslash A$. We shall show the existence of a ball $B_{i}$ from (ii).

Applying Lemma 1 below, we can assume that all the generalized principal curvatures $k_{i}(a, n), i=1, \ldots, d-1$, exist and are finite at $(a, n)$. A simple argument of geometric measure theory yields that for almost all $(a, n), k_{i}(a, n), i=1, \ldots, d-1$, are generalized principal curvatures of some of the sets $\bigcap_{I} A_{i}$ at $(a, n)$ with fulldimensional tangent cone at $a$. The proof is finished by applying Lemma 2 below.

Lemma 1. Let $A \in \mathcal{U}_{P R}$ and denote by nor* $A$ the set of all $(a, n) \in$ nor $A$ such that the generalized principal curvatures $k_{i}(a, n), i=1, \ldots, d-1$, exist and are finite. Then

$$
\mathcal{H}^{d-1}\left(\partial A \backslash \pi_{0}\left(\text { nor }^{*} A\right)\right)=0
$$

where $\pi_{0}$ is the coordinate projection $(a, n) \mapsto a$.

Proof. The generalized principal curvatures $k_{i}(a, n), i=1, \ldots, d-1$, exist at almost all $(a, n) \in$ nor $A$ by [16, Proposition 2.4]. It follows also from the result mentioned that the $(d-1)$-dimensional Jacobian of $\pi_{0}$ restricted to nor $A$ vanishes at points where at least one of the generalized principal curvatures is infinite. The assertion follows hence by the area theorem $[2, \S 3.2 .22]$.

Lemma 2. Assume that reach $A>0$ and that the generalized principal curvatures $k_{i}(a, n), i=1, \ldots, d-1$, exist and are finite at a point $(a, n) \in$ nor $A$ such that $(a,-n) \notin$ nor $A$. Then there exists an $\varepsilon>0$ such that $B(a-\varepsilon n, \varepsilon) \subseteq A$. 
Proof. Fix some $0<s<\operatorname{reach} A$ and consider the parallel set $A_{s}$ which has $C^{1,1}$ smooth boundary. The (classical) principal curvatures of $\partial A_{s}$ at $b=a+s n$ exist and equal

$$
k_{i}^{s}(b)=\frac{k_{i}(a, n)}{1+s k_{i}(a, n)}, \quad i=1, \ldots, d-1
$$

(see [24]). Denote $K=\max \left\{0, \max _{1 \leq i \leq d-1} k_{i}(a, n)\right\}$; we have

$$
k_{i}^{s}(b) \leq \frac{K}{1+s K}<\frac{1}{s}, \quad i=1, \ldots, d-1 .
$$

It follows from the basic differential calculus that, taking $s<t<s+\frac{1}{K}$, there exists a $\tau>0$ such that $B(b-t n, t) \cap B(b, \tau) \subseteq A_{s}$. Since clearly $B(a, s) \cap \partial A_{s}=\{b\}$, the distance of $B(a, s) \backslash B(b, \tau)$ from $\partial A_{s}$ is positive and, therefore, there exists an $\varepsilon>0$ with $B(a-\varepsilon n, s+\varepsilon) \subseteq A_{s}$. It follows that $B(a-\varepsilon n, \varepsilon) \subseteq A$, which completes the proof.

Proposition 2. Any topologically regular set from the convex ring is gentle.

Proof. Let $A=\bigcup_{i} A_{i}$ be a topologically regular set from the convex ring, with convex bodies $A_{i}$. We shall first versify property (i). Since $N(\partial A)=N(A) \cup N\left(A^{*}\right)$ and it is well known that $N(A)$ has locally finite $(d-1)$-dimensional Hausdorff measure, is is sufficient that the same holds for $N\left(A^{*}\right)$. We shall show that

$$
(a, n) \in N\left(A^{*}\right) \Longrightarrow(a,-n) \in \operatorname{nor} \bigcap_{i \in I} A_{i}
$$

for the index set $I=\left\{i: a \in A_{i}\right\}$. Since all the unit normal bundles nor $\bigcap_{i \in I} A_{i}$ have locally finite $(d-1)$-dimensional Hausdorff measure, (i) will follow.

Assume, for the contrary, that (6) is violated by some $(a, n) \in N\left(A^{*}\right)$. We have by definition $B(a+\delta n, \delta) \subset A$ for some $\delta>0$ and we can assume without loss of generality that even $B(a+\delta n, \delta) \subset \bigcup_{i \in I} A_{i}$ for $I=\left\{i: a \in A_{i}\right\}$. As $(a,-n) \notin$ nor $\bigcap_{i \in I} A_{i}$, there exists a point $b \in \bigcap_{i \in I} A_{i}$ with $(b-a) \cdot(-n)>0$. From the convexity of the sets $A_{i}$ it follows easily that the convex hull of $B(a+\delta n, \delta) \cup\{b\}$ is included in $\bigcup_{i \in I} A_{i}$. But as $a$ is an interior point of the convex hull of $B(a+\delta n, \delta) \cup$ $\{b\}, a$ is an interior point of $A$ as well, which is a contradiction.

In order to verify (ii), it will be sufficient to show that $A$ is full-dimensional and apply Proposition 1. As topological regularity and full dimensionality are local properties, we may assume $A=\bigcup_{i=1}^{m} A_{i}$, where $A_{1}, \ldots, A_{m}$ are convex bodies. For a fixed $a \in A$, we may assume that $a \in A_{1} \cap \ldots \cap A_{k}$ and $a \notin A_{k+1} \cup \ldots \cup A_{m}$ for some $k \in\{1, \ldots, m\}$. As $a \in \overline{\operatorname{int} A}$, there is a non-empty open ball $B$ contained in $A$ which does not hit $A_{k+1} \cup \ldots \cup A_{m}$ and thus $B \subseteq A_{1} \cup \ldots \cup A_{k}$. Therefore at least one of the sets $A_{1}, \ldots, A_{k}$ must have an interior point. As $a \in A$ was arbitrary, this shows $A=\bigcup_{i=1, \operatorname{int} A_{i} \neq \emptyset}^{m} A_{i}$. A convex body with interior points is full-dimensional, and thus, $A$ is full-dimensional, too.

We conjecture that any full-dimensional $\mathcal{U}_{P R}$ set fulfills (i). Currently we are able to prove (i) for $\mathcal{U}_{P R^{-}}$sets satisfying an additional condition. We say that sets $A_{1}, \ldots, A_{k}(k \geq 2)$ with positive reach osculate if there exists a point $a \in \bigcap_{i=1}^{k} A_{i}$ and outer normal vectors $n_{i} \in \operatorname{Nor}\left(A_{i}, a\right)$, not all of them being the zero vector, such that $\sum_{i=1}^{k} n_{i}=o$. Note that the last condition is equivalent to that the convex cone $\sum_{i=1}^{n} \operatorname{Nor}\left(A_{i}, a\right)$ contains a line. Further, we say that $A=\bigcup_{i} A_{i}$ is a non-osculating $\mathcal{U}_{P R}$ representation provided that it is a $\mathcal{U}_{P R}$ representation and the sets $A_{i}, i \in I$ do 
not osculate for any finite index set $I$ (cf. [16]). Note that any $\mathcal{U}_{P R}$ set formed by a stationary isotropic process of particles with positive reach admits a non-osculating $\mathcal{U}_{P R}$ representation (see [16, Remark 3.2]).

Proposition 3. Any $\mathcal{U}_{P R^{-}}$set admitting a non-osculating $\mathcal{U}_{P R}$ representation satisfies (i).

Proof. Let $A=\bigcup_{i} A_{i}$ be a non-osculating $\mathcal{U}_{P R}$ representation. As in the proof of Proposition 2, it will be sufficient to verify (6). If (6) were not true then there would be a pair $(a, n) \in N\left(A^{*}\right)$ and a vector $v \in \operatorname{Tan}\left(\bigcap_{i \in I} A_{i}, a\right)$ with $v \cdot(-n)>0$, where $I=\left\{i: a \in A_{i}\right\}$. Then, since $A$ contains a ball $B(a+\delta n, \delta)$ for some $\delta>0$, $\bigcup_{i \in I} \operatorname{Tan}\left(A_{i}, a\right)=\mathbb{R}^{d}$. Since the sets $A_{i}, i \in I$, do not osculate, the convex cone $\sum_{i=1}^{n} \operatorname{Nor}\left(A_{i}, a\right)$ contains no line. Hence, there is a vector, say $u$, forming an acute angle with any vector from $\sum_{i \in I} \operatorname{Nor}\left(A_{i}, a\right)$. Then, of course, $u$ cannot be tangent to any of the sets $A_{i}$, a contradiction.

A main tool for our considerations will be a far-reaching generalization of Steiner's formula, taken from Theorem 2.1 in [9], applied to $\partial A$ (see also [9, Theorem 5.2]). Here we use that $\mathcal{H}^{d}(\partial A)=0$ if $A$ is gentle. Let $\kappa_{k}$ denote the volume of the unit ball in $\mathbb{R}^{k}$.

Proposition 4. If $A$ is a closed gentle set then there are uniquely determined signed measures $\mu_{0}(\partial A, \cdot), \ldots, \mu_{d-1}(\partial A, \cdot)$ on $\mathbb{R}^{d} \times \mathbb{S}^{d-1}$, vanishing outside $N(\partial A)$, with the following property:

For any measurable bounded function $f$ on $\mathbb{R}^{d}$ with compact support, we have

$$
\int_{\mathbb{R}^{d}} f d \mathcal{H}^{d}=\sum_{i=1}^{d} i \kappa_{i} \int_{N(\partial A)} \int_{0}^{\delta(\partial A ; a, n)} t^{i-1} f(a+t n) d t \mu_{d-i}(\partial A ; d(a, n)) .
$$

The signed measures $\mu_{0}(\partial A, \cdot), \ldots, \mu_{d-1}(\partial A, \cdot)$ are called support measures of $\partial A$ and have locally finite total variation (due to [9, Corollary 2.5 and (2.13)] and the assumption $\left.\mathcal{H}^{d-1}(N(\partial A))<\infty\right)$, which allows to avoid the notion of reach measures. The support measure of order $(d-1)$ is closely related to the $(d-1)$-dimensional Hausdorff-measure on $\partial A$. [9, Proposition 4.1 and Proposition 5.1] and (5) give

$$
\mu_{d-1}(\partial A ; \cdot)=\frac{1}{2} \int_{\partial A} \mathbf{1}_{\{(a, n(A ; a)) \in \cdot\}}+\mathbf{1}_{\{(a,-n(A ; a)) \in \cdot\}} d \mathcal{H}^{d-1}(a),
$$

where the function $n(A ; \cdot)$ was defined after the introduction of gentle sets. Hence,

$$
2 \mu_{d-1}(\partial A ; \cdot)=C_{d-1}(A, \cdot)+C_{d-1}^{*}(A, \cdot),
$$

where $C_{d-1}^{*}(A, \cdot)$ is the image measure of $C_{d-1}(A, \cdot)$ under the reflection $(a, n) \mapsto$ $(a,-n)$.

\section{Dilation- AND erosion-Volumes structured by COMPACT SETS}

Given a compact subset $M$ of $\mathbb{R}^{d}$, denote $\check{M}=\{-x: x \in M\}$ and let

$$
h(M, u)=h(\operatorname{conv} M, u)=\sup \{y \cdot u: y \in M\}
$$

be the support function of (the convex hull of) $M$. 
Theorem 1. Let $A$ be a closed gentle set, $C \subset \mathbb{R}^{d}$ a bounded Borel set and $B, W$ and $P, Q$ four non-empty compact subsets of $\mathbb{R}^{d}$. Then

$$
\begin{aligned}
\lim _{\varepsilon \rightarrow 0_{+}} & \frac{1}{\varepsilon} \mathcal{H}^{d}\left(\xi_{\partial A}^{-1}(C) \cap[(A \oplus \varepsilon P) \ominus \varepsilon B] \backslash[(A \ominus \varepsilon Q) \oplus \varepsilon W]\right) \\
& =2 \int_{N(A)} \mathbf{1}_{C}(a)(h(P \oplus \check{Q}, n)-h(\check{B} \oplus W, n))^{+} C_{d-1}(A ; d(a, n)) .
\end{aligned}
$$

Proof. It can be assumed without loss of generality that $B \cup W \cup P \cup Q$ is contained in the ball $B(o, 1 / 2)$. For $\varepsilon>0$, we set

$$
g_{\varepsilon}(z)=\mathbf{1}_{\{z+\varepsilon \check{B} \subseteq A \oplus \varepsilon P\}} \mathbf{1}_{\left\{z+\varepsilon \check{W} \subseteq \mathbb{R}^{d} \backslash(A \ominus \varepsilon Q)\right\}}
$$

and apply Proposition 4 to the function

$$
f(z)= \begin{cases}\mathbf{1}_{C}\left(\xi_{\partial A}(z)\right) g_{\varepsilon}(z), & z \notin \operatorname{exo}(\partial A) \\ 0, & z \in \operatorname{exo}(\partial A) .\end{cases}
$$

Clearly,

$$
\mathcal{H}^{d}\left(\xi_{\partial A}^{-1}(C) \cap[(A \oplus \varepsilon P) \ominus \varepsilon B] \backslash[(A \ominus \varepsilon Q) \oplus \varepsilon W]\right)=\int_{\mathbb{R}^{d}} f d \mathcal{H}^{d}
$$

is the left hand side of (7). We shall show that on the right hand side, all the summands with $i>1$ are of order $o(\varepsilon)$ as $\varepsilon \rightarrow 0_{+}$, so that

$$
\begin{aligned}
\lim _{\varepsilon \rightarrow 0_{+}} & \frac{1}{\varepsilon} \mathcal{H}^{d}\left(\xi_{\partial A}^{-1}(C) \cap[(A \oplus \varepsilon P) \ominus \varepsilon B] \backslash[(A \ominus \varepsilon Q) \oplus \varepsilon W]\right) \\
& =2 \lim _{\varepsilon \rightarrow 0_{+}} \int_{N(\partial A)} \mathbf{1}_{C}(a) \int_{0}^{\delta(\partial A ; a, n)} \frac{g_{\varepsilon}(a+t n)}{\varepsilon} d t \mu_{d-1}(\partial A ; d(a, n)) \\
& =2 \lim _{\varepsilon \rightarrow 0_{+}} \int_{N(A)} \mathbf{1}_{C}(a) G_{\varepsilon}(a, n) C_{d-1}(A ; d(a, n)),
\end{aligned}
$$

where we have used $(8), N(A) \subseteq N(\partial A)$ and the abbreviation

$$
G_{\varepsilon}(a, n)=\int_{-\delta(\partial A ; a,-n)}^{\delta(\partial A ; a, n)} \frac{g_{\varepsilon}(a+t n)}{\varepsilon} d t .
$$

Indeed, $\left|g_{\varepsilon}\right|$ is bounded by 1 and since $B \neq \emptyset$ and $P$ are contained in $B(o, 1 / 2)$, the support of $g_{\varepsilon}$ is contained in $A \oplus B(o, \varepsilon)$. Hence we have

$$
\begin{aligned}
& \left|\int_{N(\partial A)} \mathbf{1}_{C}(a) \int_{0}^{\delta(\partial A ; a, n)} t^{i-1} \frac{g_{\varepsilon}(a+t n)}{\varepsilon} d t \mu_{d-i}(\partial A ; d(a, n))\right| \\
& \leq \frac{1}{\varepsilon} \int_{0}^{\varepsilon} \int_{N(\partial A)} \mathbf{1}_{C}(a) t^{i-1}\left|\mu_{d-i}\right|(\partial A ; d(a, n)) d t \\
& \quad=\frac{\varepsilon^{i-1}}{i}\left|\mu_{d-i}\right|\left(\partial A ; C \times \mathbb{S}^{d-1}\right)
\end{aligned}
$$

and the total variation measure $\left|\mu_{d-i}\right|\left(\partial A ; C \times \mathbb{S}^{d-1}\right)$ is finite for any $i$ since $C$ is bounded, thus the last expression tends to 0 with $\varepsilon \rightarrow 0_{+}$whenever $i>1$.

It remains to show that

$$
G_{\varepsilon}(a, n) \rightarrow(h(P \oplus \check{Q}, n)-h(\check{B} \oplus W, n))^{+}, \quad \varepsilon \rightarrow 0_{+},
$$


for $C_{d-1}(A ; \cdot)$-almost all $(a, n)$, and apply the Lebesgue dominated theorem. As $A$ is gentle and by the definition of $C_{d-1}(A, \cdot), C_{d-1}(A ; \cdot)$-almost all $(a, n) \in N(A)$ satisfy $\delta_{+}:=\delta(A ; a, n)>0$ and $\delta_{-}:=\delta(A ; a,-n)>0$. Fix such a pair $(a, n) \in N(A)$ and assume $\varepsilon<\min \left\{\delta_{+}, \delta_{-}\right\} / 2$. It follows from the definitions of $\delta_{+}$and $\delta_{-}$that $A$ has no points inside the ball $B_{o}$ of center $a+\delta_{+} n$ and radius $\delta_{+}$, and that the ball $B_{i}$ of center $a-\delta_{-} n$ and radius $\delta_{-}$is a subset of $A$. Fix $p \in P$ with $p \cdot n=h(P, n)$ and $q \in Q$ with $q \cdot(-n)=h(Q,-n)$. As $\mathbb{R}^{d} \backslash(A \ominus \varepsilon Q)=\left(\mathbb{R}^{d} \backslash A\right) \oplus \varepsilon Q$, we have

$$
g_{\varepsilon}(z) \geq \mathbf{1}_{\left\{z+\varepsilon \check{B} \subseteq B_{i}+\varepsilon p\right\}} \mathbf{1}_{\left\{z+\varepsilon \check{W} \in \operatorname{int} B_{o}+\varepsilon q\right\}} .
$$

From the definition of the support function, and since $\check{B}-p$ lies in the unit ball, the set $\varepsilon \check{B}-\varepsilon p$ is contained in the ball $B(o, \varepsilon)$ intersected with the half-space $\{y: y \cdot n \leq \varepsilon h(\check{B}, n)-\varepsilon h(P, n)\}$. If $z=a+t n$ and $-\delta_{-}<t<-\varepsilon h(\check{B}, n)+\varepsilon h(P, n)-$ $\left(\delta_{-}-\sqrt{\delta_{-}^{2}-\varepsilon^{2}}\right)$ then the first indicator function in (12) is 1. Similarly, the second indicator function is 1 , if $z=a+t n$ and $\varepsilon h(\check{W},-n)-\varepsilon h(Q,-n)+\left(\delta_{+}-\sqrt{\delta_{+}^{2}-\varepsilon^{2}}\right)<$ $t<\delta_{+}$; thus (12) implies

$$
\begin{aligned}
& G_{\varepsilon}(a, n) \geq(-h(\check{B}, n)+h(P, n)-h(W, n)+h(\check{Q}, n) \\
&\left.-\varepsilon^{-1}\left(\delta_{-}-\sqrt{\delta_{-}^{2}-\varepsilon^{2}}\right)-\varepsilon^{-1}\left(\delta_{+}-\sqrt{\delta_{+}^{2}-\varepsilon^{2}}\right)\right)^{+} \\
& \rightarrow(h(P \oplus \check{Q}, n)-h(\check{B} \oplus W, n))^{+}, \quad \varepsilon \rightarrow 0_{+} .
\end{aligned}
$$

Let $w \in \check{W}$ and $b \in \check{B}$ be such that $w \cdot(-n)=h(\check{W},-n)$ and $b \cdot n=h(\check{B}, n)$. Using $A \oplus \varepsilon P=\mathbb{R}^{d} \backslash\left(\left(\mathbb{R}^{d} \backslash A\right) \ominus \varepsilon P\right)$, we see that

$$
g_{\varepsilon}(z) \leq \mathbf{1}_{\left\{z+\varepsilon b+\varepsilon \check{P} \subseteq \operatorname{int} B_{o}\right\}} \mathbf{1}_{\left\{z+\varepsilon w+\varepsilon \check{Q} \subseteq B_{i}\right\}} .
$$

Similar arguments as above yield the upper bound

$$
\begin{aligned}
& G_{\varepsilon}(a, n) \leq(-h(\check{B}, n)+h(P, n)-h(W, n)+h(\check{Q}, n) \\
&\left.+\varepsilon^{-1}\left(\delta_{-}-\sqrt{\delta_{-}^{2}-\varepsilon^{2}}\right)+\varepsilon^{-1}\left(\delta_{+}-\sqrt{\delta_{+}^{2}-\varepsilon^{2}}\right)\right)^{+} \\
& \rightarrow(h(P \oplus \check{Q}, n)-h(\check{B} \oplus W, n))^{+}, \quad \varepsilon \rightarrow 0_{+} .
\end{aligned}
$$

The proof is completed by applying the Lebesgue dominated theorem: The fact that $g_{\varepsilon}(z) \leq \mathbf{1}_{(A \oplus B(0, \varepsilon)) \cap\left(A^{*} \oplus B(0, \varepsilon)\right.}(z)$ implies

$$
0 \leq \mathbf{1}_{C}(a) G_{\varepsilon}(a, n) \leq \mathbf{1}_{C}(a) \frac{1}{\varepsilon} \int_{-\delta(\partial A ; a,-n)}^{\delta(\partial A ; a, n)} \mathbf{1}_{\{-\varepsilon \leq t \leq \varepsilon\}} d t \leq 2 \cdot \mathbf{1}_{C}(a),
$$

which yields a uniformly integrable upper bound.

We give some particular cases of Theorem 1.

Corollary 1. Let $A$ be a closed gentle set and $C$ a bounded Borel set. Fix nonempty compact subsets $B, W$ and $Q$ of $\mathbb{R}^{d}$. Then 
(1) $\lim _{\varepsilon \rightarrow 0_{+}} \frac{1}{\varepsilon} \mathcal{H}^{d}\left(\xi_{\partial A}^{-1}(C) \cap[A \oplus \varepsilon Q] \backslash A\right)$

$$
\begin{aligned}
& =\int_{N(A)} \mathbf{1}_{C}(a) h^{+}(Q, n) C_{d-1}(A, d(a, n)) \\
& =\int_{N(A)} \mathbf{1}_{C}(a) h(Q \cup\{0\}, n) C_{d-1}(A, d(a, n)),
\end{aligned}
$$

(2) $\lim _{\varepsilon \rightarrow 0_{+}} \frac{1}{\varepsilon}\left(\mathcal{H}^{d}\left(\xi_{\partial A}^{-1}(C) \cap[A \oplus \varepsilon Q]\right)-\mathcal{H}^{d}\left(\xi_{\partial A}^{-1}(C) \cap A\right)\right)$

$$
=\int_{N(A)} \mathbf{1}_{C}(a) h(Q, n) C_{d-1}(A, d(a, n))
$$

(3) $\lim _{\varepsilon \rightarrow 0_{+}} \frac{1}{\varepsilon} \mathcal{H}^{d}\left(\xi_{\partial A}^{-1}(C) \cap[A \ominus \varepsilon B] \backslash[A \oplus \varepsilon W]\right)$

$$
=\int_{N(A)} \mathbf{1}_{C}(a)(-h(\check{B} \oplus W, n))^{+} C_{d-1}(A, d(a, n)) .
$$

To show Corollary 1.(2), we used

$$
\mathcal{H}^{d}(D)-\mathcal{H}^{d}(E)=\mathcal{H}^{d}(D \backslash E)-\mathcal{H}^{d}(E \backslash D)
$$

with $D=\xi_{\partial A}^{-1}(C) \cap[A \oplus \varepsilon Q]$ and $E=\xi_{\partial A}^{-1}(C) \cap A$ and applied Theorem 1. For a compact gentle set $A$, the image measure $S_{d-1}(A, \cdot)$ of $2 C_{d-1}(A, \cdot)$ under the projection $(a, n) \mapsto n$ is finite. This measure is called surface area measure of $A$ (of order $d-1)$.

Corollary 2. Let $A$ be a compact gentle set and $B, W$ and $Q$ nonempty compact subsets of $\mathbb{R}^{d}$. Then

(1) $\lim _{\varepsilon \rightarrow 0_{+}} \frac{1}{\varepsilon} \mathcal{H}^{d}([A \oplus \varepsilon Q] \backslash A)=\int_{\mathbb{S}^{d-1}} h^{+}(Q, n) S_{d-1}(A, d n)$

$$
=\int_{\mathbb{S}^{d-1}} h(Q \cup\{0\}, n) S_{d-1}(A, d n),
$$

(2) $\lim _{\varepsilon \rightarrow 0_{+}} \frac{1}{\varepsilon}\left(\mathcal{H}^{d}(A \oplus \varepsilon Q)-\mathcal{H}^{d}(A)\right)=\int_{\mathbb{S}^{d-1}} h(Q, n) S_{d-1}(A, d n)$,

(3) $\lim _{\varepsilon \rightarrow 0_{+}} \frac{1}{\varepsilon} \mathcal{H}^{d}([A \ominus \varepsilon B] \backslash[A \oplus \varepsilon W])=\int_{\mathbb{S}^{d-1}}(-h(\check{B} \oplus W, n))^{+} S_{d-1}(A, d n)$.

In [15, Theorem 3], Corollary 2.(1) was shown for certain compact sets $A \in \mathcal{U}_{P R}$ and convex bodies $P$. In the planar case, Corollary 2.(3) was shown in [10] for topologically regular sets in the convex ring and finite sets $B$ and $W$. Notice that

$$
(-h(\check{B} \oplus W, \cdot))^{+}=h(\{o\} \cup(\check{B} \oplus W), \cdot)-h(\check{B} \oplus W, \cdot)
$$

can be written as difference of support functions.

We finish this section with a uniqueness result concerning the integrals in Corollary 2.(3). It implies that the left hand side of Corollary 2.(3) determines $S_{d-1}(A, \cdot)$ uniquely, if known e.g. for all finite sets $B$ and $W$ with $\#(\check{B} \oplus W) \leq 3$, or for all pairs $(B, W)$, where one set is a singleton and the other is a (convex) triangle.

Proposition 5. Let $\mu$ and $\mu^{\prime}$ be two Borel-measures on $S^{d-1}$ such that

$$
\int_{S^{d-1}}(-h(M, n))^{+} \mu(d n)=\int_{S^{d-1}}(-h(M, n))^{+} \mu^{\prime}(d n)
$$


for all $M \subset \mathbb{R}^{d}$ with at most three points. Then $\mu=\mu^{\prime}$.

Proof. As $h(M, \cdot)=h(\operatorname{conv} M, \cdot)$, we may assume that $M$ runs through the family of (convex) triangles. Let $C$ be an arbitrary non-degenerate triangle with the property that

$$
\mu\left(\operatorname{Nor}(C, e) \cap S^{d-1}\right)=\mu^{\prime}\left(\operatorname{Nor}(C, e) \cap S^{d-1}\right)=0
$$

for all edges $e$ of $C$, where $\operatorname{Nor}(C, e)$ is the normal cone of $C$ at a relative interior point of $e$. Note that $\operatorname{Nor}(C, e)$ is a (relative open) half great sub-sphere. Let $v_{1}, v_{2}, v_{3}$ be the vertices of $C$ and let $N_{i}=\operatorname{Nor}\left(C, v_{i}\right)$. Fix $i \in\{1,2,3\}$. We have

$$
\frac{1}{\varepsilon}\left(-h\left(C-(1-\varepsilon) v_{i}, n\right)\right)^{+} \rightarrow\left\{\begin{array}{cc}
-v_{i} \cdot n, & \text { if } n \in N_{i} \\
0, & \text { if } n \notin N_{i}
\end{array}\right.
$$

as $\varepsilon \rightarrow 0_{+}$and the left hand side is bounded by $\left(-v_{i} \cdot n\right)^{+}$, so (13) with $M=$ $C-(1-\varepsilon) v_{i}$ and the Lebesgue dominated theorem imply

$$
\int_{N_{i}} v_{i} \cdot n \mu(d n)=\int_{N_{i}} v_{i} \cdot n \mu^{\prime}(d n) .
$$

If $v \in \mathbb{R}^{2}$ is arbitrary, we can chose three triangles $C_{1}, C_{2}, C_{3}$ in a two-dimensional plane parallel the affine hull of $C$ with $v$ as a vertex and $\operatorname{Nor}\left(C_{i}, v\right)=N_{i}$. Applying (15) to all three of these triangles with $v_{i}=v$ implies

$$
\int_{S^{d-1}} v \cdot n \mu(d n)=\int_{S^{d-1}} v \cdot n \mu^{\prime}(d n)
$$

and hence

$$
\int_{S^{d-1}} n \mu(d n)=\int_{S^{d-1}} n \mu^{\prime}(d n) .
$$

This implies that $\mu-\mu^{\prime}$ can be written as difference of two surface area measures by Minkowski's existence theorem; see e.g. [18, Theorem 7.1.2.].

Now fix an arbitrary triangle $T$ in a plane $L$. Circumscribe a triangle $C \subset L$ with vertices $v_{1}, v_{2}, v_{3} \notin T$ such that (14) holds. Recall that $N_{i}=\operatorname{Nor}\left(C, v_{i}\right)$. We have

$$
\left(-h\left(T-v_{i}, n\right)\right)^{+}=-\mathbf{1}_{N_{i}}(n) h\left(T-v_{i}, n\right)=-\mathbf{1}_{N_{i}}(n)\left(h(T, n)-v_{i} \cdot n\right) .
$$

As integration of this equality with $\mu$ and $\mu^{\prime}$ leads to the same result, (15) implies

$$
\int_{N_{i}} h(T, n) \mu(d n)=\int_{N_{i}} h(T, n) \mu^{\prime}(d n) .
$$

Summation over $i=1,2,3$ yields

$$
\int_{S^{d-1}} h(T, n)\left(\mu-\mu^{\prime}\right)(d n)=0
$$

for all triangles $T$. As $\mu-\mu^{\prime}$ can be written as a difference of surface area measures, a theorem of Schneider [17] (see also [18, p. 283]) implies $\mu=\mu^{\prime}$, as required. 


\section{VOLUME-THRESHOLD SETS}

We turn to volume-threshold sets. Recall the definition

$$
A \oslash_{\theta} Q=\left\{x \in \mathbb{R}^{d}: \mathcal{H}^{d}(A \cap(x+\check{Q})) \geq \theta \mathcal{H}^{d}(Q)\right\}
$$

where $0<\theta \leq 1, A$ and $Q$ are Borel sets in $\mathbb{R}^{d}$ and $0<\mathcal{H}^{d}(Q)<\infty$. To introduce a function, which takes the place of support functions in asymptotic results, some further notation is needed. Let $H_{r, n}$ be the half space

$$
H_{r, n}=\left\{x \in \mathbb{R}^{d}: x \cdot n \leq r\right\} .
$$

It was noted in [4], that the function

$$
\begin{aligned}
f_{\theta}(Q, n) & =\sup \left\{r \in \mathbb{R}: \mathcal{H}^{d}\left(H_{-r, n} \cap Q\right) \geq \theta \mathcal{H}^{d}(Q)\right\} \\
& =-\inf \left\{r \in \mathbb{R}: \mathcal{H}^{d}\left(H_{r, n} \cap Q\right) \geq \theta \mathcal{H}^{d}(Q)\right\}, \quad n \in \mathbb{S}^{d-1},
\end{aligned}
$$

has the symmetry property

$$
f_{1-\theta}(Q, n)=-f_{\theta}(Q,-n)
$$

if $0<\theta<1$. It is also positively homogeneous, i.e. $f_{\theta}(\varepsilon Q, n)=\varepsilon f_{\theta}(Q, n)$ for $\varepsilon>0$. We mention that in the case of a convex body $Q$ with sufficiently smooth boundary and if $\theta<1$ is close enough to 1 , the function $-f_{\theta}(Q, \cdot)$ is the support function of a convex body. This convex body is called floating body of $Q$, see e.g. [23] for details. For $\theta=1$, we have the following: if $Q \neq \emptyset$ is a topologically regular compact set but otherwise arbitrary, then $-f_{1}(Q, \cdot)=h(Q, \cdot)$. More generally, consider an arbitrary compact set $Q$ with positive volume. It is easy to see that the positive homogeneous extension of the function

$$
n \mapsto-f_{1}(Q, n)=\inf \left\{r \in \mathbb{R}^{d} \mid \mathcal{H}^{d}\left(H_{r, n} \cap \check{Q}\right)=0\right\}
$$

is convex. [18, Theorem 1.7.1] implies that there is a convex body with support function $-f_{1}(Q, \cdot)$. The intersection $\tilde{Q}$ of this convex body with $Q$ satisfies $\mathcal{H}^{d}(\tilde{Q})=$ $\mathcal{H}^{d}(Q)$ and thus

$$
D \oslash_{1} Q=D \ominus \tilde{Q} \text { and }-f_{1}(Q, \cdot)=h(\tilde{Q}, \cdot),
$$

where $D \subseteq \mathbb{R}^{d}$ is an arbitrary Borel set. This will be used later.

Gentle sets $A$ have the property that in the neighborhood of almost every boundary point $a$, the sets $A$ and $A^{*}$ can be approximated by balls. These balls in turn can be approximated by half spaces with $a$ in their boundary, if only the local volume is of interest. The following Lemma shows the asymptotic behavior of such an approximation for a given ball. It can either be shown by direct calculation or derived from more general results (see the remarks in [4, p. 1525]).

Lemma 3. Let $B \subset \mathbb{R}^{d}$ be a ball (with positive radius) and $a \in \partial B$. Let $n$ be the outer unit normal of $B$ at $a$ and define the local volume deficit

$$
\gamma(B, a ; \varepsilon):=\mathcal{H}^{d}\left(B(a, \varepsilon) \cap\left(H_{a \cdot n, n} \backslash B\right)\right) .
$$

Then $\gamma(B, a ; \varepsilon)=o\left(\varepsilon^{d}\right)$ as $\varepsilon \rightarrow 0_{+}$. 
Theorem 2. Fix $0<\theta \leq 1,0<\varrho \leq 1$ and a closed gentle set $A$. Let the measurable set $C \subset \mathbb{R}^{d}$ be bounded. Let $B, W, P, Q \subset \mathbb{R}^{d}$ be non-empty compact sets and assume $\mathcal{H}^{d}(P)>0$ and $\mathcal{H}^{d}(Q)>0$. Then

$$
\begin{aligned}
\lim _{\varepsilon \rightarrow 0_{+}} & \frac{1}{\varepsilon} \mathcal{H}^{d}\left(\xi_{\partial A}^{-1}(C) \cap\left[\left(A \oslash_{\theta} \varepsilon P\right) \ominus \varepsilon B\right] \backslash\left[\left(A \oslash_{\varrho} \varepsilon Q\right) \oplus \varepsilon W\right]\right) \\
\quad & =2 \int_{N(A)} \mathbf{1}_{C}(a)\left(f_{\theta}(\check{P}, n)-f_{\varrho}(\check{Q}, n)-h(\check{B} \oplus W, n)\right)^{+} C_{d-1}(A ; d(a, n)) .
\end{aligned}
$$

Proof. Consider first the case, where $\theta<1$ and $\varrho<1$. The cases where one or both of these parameters are 1 will be discussed at the end of this proof. We may assume that $B \cup W \cup P \cup Q \subseteq B(o, 1 / 2)$ holds. Put

$$
g_{\varepsilon}(z)=\mathbf{1}_{\left\{z+\varepsilon \check{B} \subseteq A \oslash_{\theta} \varepsilon P\right\}} \mathbf{1}_{\left\{z+\varepsilon \check{W} \subseteq \mathbb{R}^{d} \backslash\left(A \oslash_{\varrho} \varepsilon Q\right)\right\}}
$$

and apply Proposition 4 with $f(z)=\mathbf{1}_{C}\left(\xi_{\partial A}(z)\right) g_{\varepsilon}(z)$, whenever $z \notin \operatorname{exo}(\partial A) \cup \partial A$ (and $f(z)=0$, otherwise). As the support of $g_{\varepsilon}$ lies in the set $A \oplus B(o, \varepsilon)$, the same arguments like in the proof of Theorem 1 imply

$$
\begin{aligned}
\lim _{\varepsilon \rightarrow 0_{+}} & \frac{1}{\varepsilon} \mathcal{H}^{d}\left(\xi_{A}^{-1}(C) \cap\left[\left(A \oslash_{\theta} \varepsilon P\right) \ominus \varepsilon B\right] \backslash\left[\left(A \oslash_{\varrho} \varepsilon Q\right) \oplus \varepsilon W\right]\right) \\
= & \lim _{\varepsilon \rightarrow 0_{+}} 2 \int_{N(A)} \mathbf{1}_{C}(a) G_{\varepsilon}(a, n) C_{d-1}(A ; d(a, n))
\end{aligned}
$$

with $G_{\varepsilon}(a, n)=\int_{-\delta(\partial A ; a,-n)}^{\delta(\partial A ; a, n)} \frac{g_{\varepsilon}(a+t n)}{\varepsilon} d t$. We show that

$$
G_{\varepsilon}(a, n) \rightarrow\left(f_{\theta}(\check{P}, n)-f_{\varrho}(\check{Q}, n)-h(\check{B} \oplus W, n)\right)^{+}, \quad \varepsilon \rightarrow 0_{+}
$$

for $C_{d-1}(A ; \cdot)$-almost all $(a, n) \in N(A)$. Let $(a, n) \in N(A)$. Like in the proof of Theorem 1 , we may assume that $\delta_{+}=\delta_{+}(A ; a, n)$ and $\delta_{-}=\delta(A ; a,-n)$ are positive radii of balls $B_{o} \subseteq A^{*}$ and $B_{i} \subseteq A$ with centers $a+\delta_{+} n$ and $a-\delta_{-} n$, respectively. Assume $\varepsilon<\min \left\{\delta_{+} / 2, \delta_{-} / 2, \theta, \varrho\right\}$. As

$$
A^{*} \oslash_{\tau} \varepsilon Q \subseteq \mathbb{R}^{d} \backslash\left(A \oslash_{\varrho} \varepsilon Q\right)
$$

holds for all $1-\varrho<\tau<1$, we have

$$
g_{\varepsilon}(z) \geq \mathbf{1}_{\left\{z+\varepsilon \check{B} \subseteq B_{i} \oslash_{\theta} \varepsilon P\right\}} \mathbf{1}_{\left\{z+\varepsilon \breve{W} \subseteq B_{o} \oslash_{1-\varrho+\varepsilon} \varepsilon Q\right\}} .
$$

We will show that the first indicator function is 1 , if $z=a+t n$ with

$$
-\delta_{-} \leq t \leq \varepsilon f_{\theta(\varepsilon)}(\check{P}, n)-\varepsilon h(\check{B}, n)
$$

where

$$
\theta(\varepsilon)=\theta+\frac{\gamma\left(B_{i}, a ; \sqrt{2} \varepsilon\right)}{\varepsilon^{d} \mathcal{H}^{d}(\check{P})} .
$$

Note that $\theta(\varepsilon) \rightarrow \theta$ according to Lemma 3 and thus $f_{\theta(\varepsilon)}(\check{P}, n) \rightarrow f_{\theta}(\check{P}, n)$, as $\varepsilon \rightarrow 0_{+}$. Let $b \in \check{B}$ be arbitrary. If (21) holds, then

$$
f_{\theta(\varepsilon)}(\varepsilon \check{P}, n) \geq t+\varepsilon h(\check{B}, n) \geq t+\varepsilon b \cdot n .
$$

In view of the definition of $f_{\theta}(\varepsilon \check{P}, \cdot)$ we obtain $\theta(\varepsilon) \mathcal{H}^{d}(\varepsilon \check{P}) \leq \mathcal{H}^{d}\left(H_{t+\varepsilon b \cdot n, n} \cap \varepsilon \check{P}\right)$, which can be rewritten as

$$
\theta \mathcal{H}^{d}(\varepsilon \check{P}) \leq \mathcal{H}^{d}\left(H_{a \cdot n, n} \cap(a+t n+\varepsilon b+\varepsilon \check{P})\right)-\gamma\left(B_{i}, a ; \sqrt{2} \varepsilon\right) .
$$


As $t n+\varepsilon b+\varepsilon \check{P}$ is contained in the cylinder $\left(B(o, \varepsilon) \cap n^{\perp}\right) \oplus\left[-\left(\delta_{-}+\varepsilon\right) n, \varepsilon n\right]$, we have $a+t n+\varepsilon b+\varepsilon \check{P} \subseteq B(a, \sqrt{2} \varepsilon) \cup B_{i}$. The definition of $\gamma\left(B_{i}, a ; \cdot\right)$ implies

$$
\theta \mathcal{H}^{d}(\varepsilon \check{P}) \leq \mathcal{H}^{d}\left(B_{i} \cap(a+t n+\varepsilon b+\varepsilon \check{P})\right)
$$

and thus $a+t n+\varepsilon b \in B_{i} \oslash_{\theta} \varepsilon P$. As $b \in \check{B}$ was arbitrary and $z=a+t n$, the first indicator function in (20) is 1.

We now show that the second indicator function in (20) is 1 for $z=a+t n$ if $\varepsilon f_{\varrho(\varepsilon)}(\check{Q}, n)+\varepsilon h(W, n) \leq t \leq \delta_{+}$, where

$$
\varrho(\varepsilon)=\varrho-\varepsilon-\frac{\gamma\left(B_{o}, a ; \sqrt{2} \varepsilon\right)}{\varepsilon^{d} \mathcal{H}^{d}(\check{Q})} .
$$

In view of (17), the condition on $t$ implies

$$
-f_{1-\varrho(\varepsilon)}(\varepsilon \check{Q},-n) \leq t+\varepsilon w \cdot n
$$

for all $w \in \check{W}$. This gives $\mathcal{H}^{d}\left(H_{t+\varepsilon w \cdot n,-n} \cap \varepsilon \check{Q}\right) \geq(1-\rho(\varepsilon)) \mathcal{H}^{d}(\varepsilon \check{Q})$ and implies

$$
\mathcal{H}^{d}\left(H_{a \cdot(-n),-n} \cap(a+t n+\varepsilon w+\varepsilon \check{Q})-\gamma\left(B_{o}, a ; \sqrt{2} \varepsilon\right) \geq(1-\rho+\varepsilon) \mathcal{H}^{d}(\varepsilon \check{Q}) .\right.
$$

As above, $a+t n+\varepsilon \check{W} \subseteq B_{o} \oslash_{1-\rho+\varepsilon} \varepsilon Q$ can be concluded and the intermediate claim is shown.

These considerations lead to the estimate

$$
\begin{aligned}
G_{\varepsilon}(a, n) & \geq\left(f_{\theta(\varepsilon)}(\check{P}, n)-h(\check{B}, n)-f_{\varrho(\varepsilon)}(\check{Q}, n)-h(W, n)\right)^{+} \\
& \rightarrow\left(f_{\theta}(\check{P}, n)-f_{\varrho}(\check{Q}, n)-h(\check{B} \oplus W, n)\right)^{+}, \quad \varepsilon \rightarrow 0_{+}
\end{aligned}
$$

An upper bound for $G_{\varepsilon}$ is obtained as follows: If $w \in \check{W}$ and $b \in \check{B}$ are such that $w \cdot(-n)=h(\check{W},-n)$ and $b \cdot n=h(\check{B}, n)$, then

$$
g_{\varepsilon}(z) \leq \mathbf{1}_{\left\{z+\varepsilon b \in B_{o} \oslash_{1-\theta+\varepsilon} \varepsilon P\right\}} \mathbf{1}_{\left\{z+\varepsilon w \in B_{i} \oslash \varepsilon Q\right\}} .
$$

Treating the indicator functions as above, yields the same asymptotic bound as in (22), but now from above. It remains to apply the Lebesgue dominated convergence theorem: From (4) it follows that $g_{\varepsilon}(z) \leq \mathbf{1}_{(A \oplus B(0, \varepsilon)) \cap\left(A^{*} \oplus B(0, \varepsilon)\right)}(z)$ and thus $\mathbf{1}_{C}(a) G_{\varepsilon}(a, n) \leq 2 \cdot \mathbf{1}_{C}(a)$ is an integrable upper bound.

If one or both of the parameters $\theta$ and $\varrho$ are 1 , the above proof does not work. If, for example, $\theta=1$, then $\theta(\varepsilon)>1$ is not a threshold parameter. In this case, however, one can replace $Q$ by $\tilde{Q}$, which satisfies (18) and adapt arguments of the proof of Theorem 1. In a similar way, the present proof can be extended to include the case $\varrho=1$.

Notice that the proof can easily be adapted to the case where either $A \oslash_{\theta} \varepsilon P$ or $A \oslash_{\varrho} \varepsilon Q$ are replaced by $A$. This implies the first two statements of the following Corollary.

Corollary 3. Let $A$ be a closed gentle set and $C \subset \mathbb{R}^{d}$ a bounded Borel set. Fix $0<\theta \leq 1$ and nonempty compact subsets $B, W$ and $Q$ of $\mathbb{R}^{d}$, where $\mathcal{H}^{d}(Q)>0$. Then 


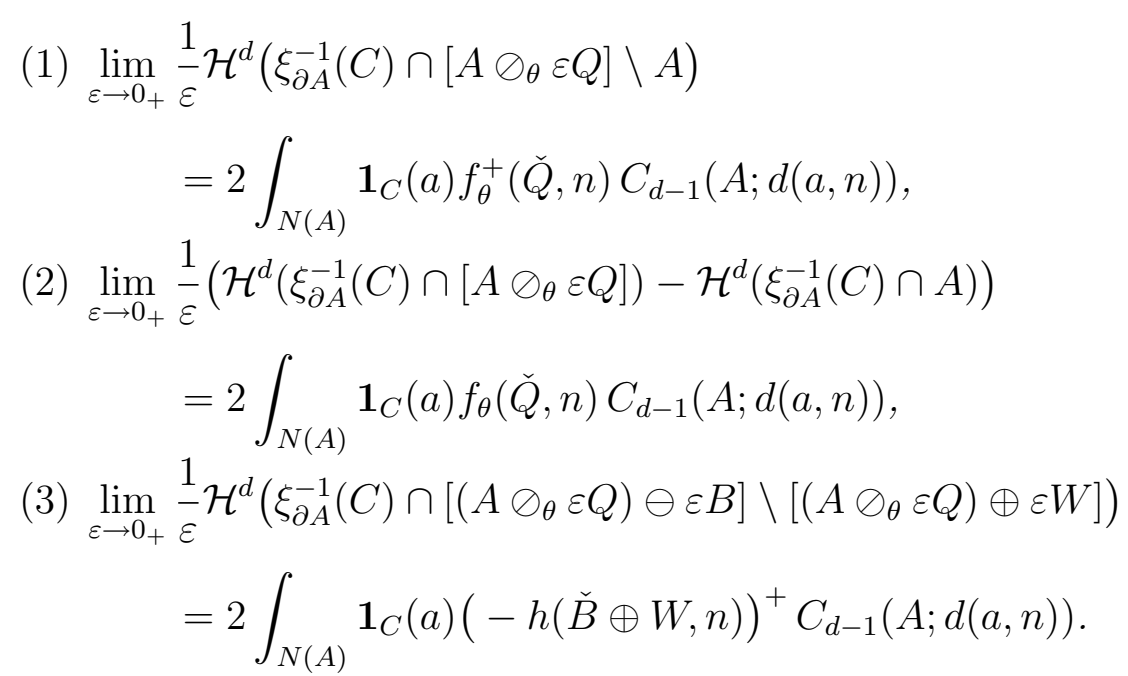

Corollary 4. Let $A$ be a compact gentle set. Fix $0<\theta \leq 1$ and nonempty compact subsets $B, W$ and $Q$ of $\mathbb{R}^{d}$, where $\mathcal{H}^{d}(Q)>0$. Then

(1) $\lim _{\varepsilon \rightarrow 0_{+}} \frac{1}{\varepsilon} \mathcal{H}^{d}\left(\left[A \oslash_{\theta} \varepsilon Q\right] \backslash A\right)=\int_{\mathbb{S}^{d-1}} f_{\theta}^{+}(\check{Q}, n) S_{d-1}(A ; d n)$,

(2) $\lim _{\varepsilon \rightarrow 0_{+}} \frac{1}{\varepsilon}\left(\mathcal{H}^{d}\left(A \oslash_{\theta} \varepsilon Q\right)-\mathcal{H}^{d}(A)\right)=\int_{\mathbb{S}^{d-1}} f_{\theta}(\check{Q}, n) S_{d-1}(A ; d n)$,

(3) $\lim _{\varepsilon \rightarrow 0_{+}} \frac{1}{\varepsilon} \mathcal{H}^{d}\left(\left[\left(A \oslash_{\theta} \varepsilon Q\right) \ominus \varepsilon B\right] \backslash\left[\left(A \oslash_{\theta} \varepsilon Q\right) \oplus \varepsilon W\right]\right)$

$$
=\int_{\mathbb{S}^{d-1}}(-h(\check{B} \oplus W, n))^{+} S_{d-1}(A ; d n) .
$$

Corollary 4.(2) was shown for convex $Q$ in [4] under considerably stronger regularity conditions on $A$. Also in this paper, $f_{\theta}(Q, \cdot)$ is explicitly determined for $d=2$ and $Q=[0,1]^{2}$. Note that the right hand side of Corollary 4.(3) coincides with the right hand side of Corollary 2.(3) and does in particular not depend on the threshold parameter, nor on $Q$.

\section{Applications in RANDOM SET THEORY}

The geometric results of the previous sections can be transferred to random closed sets. For a general introduction to the theory of random sets, see the books [20] and [21]. In the following, we consider random closed sets $Z$ which are almost surely gentle sets, but we do not assume stationarity. An integrability condition will be needed and we require that condition (i) in the definition of gentle sets does not only hold almost surely, but in mean:

$$
\mathbb{E} \mathcal{H}^{d}\left(N(\partial Z) \cap D \times \mathbb{S}^{d-1}\right)<\infty
$$

for all bounded Borel sets $D \subset \mathbb{R}^{d}$. If $Z$ is stationary (or homogeneous), i.e. the distribution of $Z$ is invariant under all translations, it is enough to require (23) for only one bounded set $D$ with positive volume. If $Z$ is a random set in the extended convex ring, the usual integrability condition

$$
\mathbb{E} 2^{N(Z \cap K)}<\infty \text { for all convex bodies } K,
$$

implies (23). Here, for a set $M \neq \emptyset$ in the convex ring, $N(M)$ is the minimal number of convex bodies needed, to represent $M$ as their union, and $N(\emptyset)=0$. 
We follow [6] and [9] to introduce the necessary terminology. If (23) holds, then

$$
\Lambda_{d-1}=\mathbb{E} C_{d-1}(Z ; \cdot)
$$

is a locally finite (positive) measure, concentrated on $\mathbb{R}^{d} \times \mathbb{S}^{d-1}$. It is called the intensity measure of $C_{d-1}(Z ; \cdot)$. A disintegration of this measure yields

$$
\Lambda_{d-1}(d(z, n))=\mathcal{R}(z, d n) \Lambda_{d-1}\left(d z \times \mathbb{S}^{d-1}\right),
$$

where $\mathcal{R}$ is a stochastic kernel from $\mathbb{R}^{d}$ to $\mathbb{S}^{d-1}$. In [6], $\mathcal{R}$ is called (position dependent) rose of direction or mean normal measure. Let

$$
\bar{p}(z)=P(z \in Z)
$$

be the volume density of $Z$ in $z \in \mathbb{R}^{d}$ and fix an arbitrary compact set $B \subset \mathbb{R}^{d}$ with $o \in B$. If $\bar{p}(z)<1$, the hit distribution function at $z$ with structuring element $B$ is defined by

$$
\begin{aligned}
H_{B}(z, t) & =P(Z \cap(z+t B) \neq \emptyset \mid z \notin Z) \\
& =P(z \in Z \oplus t \check{B} \mid z \notin Z) .
\end{aligned}
$$

For $\bar{p}(z)=1$, we set $H_{B}(z, t)=1$. Usually, $B$ is assumed to be convex and to have the origin in its interior. We do only require compactness and the weak condition $o \in B$. The latter is not needed for the general definition, but it should be adopted for reasons of intuition, as it implies $z \in z+t B$, so $z$ can be seen as a "center" of $z+t B$. The contact distribution function at $z$ is defined as

$$
\tilde{H}_{B}(z, t)=P\left(d_{B}(z) \leq t \mid z \notin Z\right),
$$

with the random variable

$$
d_{B}(z)=\min \{r \geq 0: Z \cap(z+r B) \neq \emptyset\} ;
$$

see e.g. Hug \& Last [6]. If $B$ is star shaped with respect to the origin, then $H_{B}(z, t)=$ $\tilde{H}_{B}(z, t)$. In the present general context, however, the latter definition is less general than (25): let

$$
\operatorname{star} B=\bigcup_{b \in B}[0, b]
$$

be the star-hull of $B$ with respect to 0 . As $d_{B}(z)=d_{\operatorname{star} B}(z)$, we have

$$
\tilde{H}_{B}(z, t)=H_{\text {starB }}(z, t) .
$$

In particular, the results for $H_{B}(z, \cdot)$ easily transfer to $\tilde{H}_{B}(z, \cdot)$.

Theorem 3. Let $B \subset \mathbb{R}^{d}$ be a non-empty compact set with $o \in B$. Let $Z$ be a.s. a gentle set such that (23) holds for all bounded Borel sets $D \subset \mathbb{R}^{d}$. If $\Lambda_{d-1}\left(\cdot \times \mathbb{S}^{d-1}\right)$ is absolutely continuous with respect to $\mathcal{H}^{d}$ with density $\lambda_{d-1}(\cdot)$, then

$$
t^{-1}(1-\bar{p}(z)) H_{B}(z, t) \mathcal{H}^{d}(d z) \stackrel{v}{\longrightarrow} 2 \lambda_{d-1}(z) \int_{\mathbb{S}^{d-1}} h(\check{B}, n) \mathcal{R}(z, d n) \mathcal{H}^{d}(d z),
$$

as $t \rightarrow 0_{+}$, where $\stackrel{v}{\longrightarrow}$ denotes the vague convergence of measures.

The proof follows closely those of Theorem 4.1 and Corollary 4.6 in [6]. We do not repeat it here, as similar arguments will be made explicit in the proof of Theorem 4 , below. Under the additional assumption that $B$ is convex and $o \in \operatorname{int} B$, Theorem 3 follows from [6, Theorem 4.1]. 
If $Z$ is stationary, then $\Lambda_{d-1}$ is invariant with respect to translations in its first component, so it has the form

$$
\left(\lambda_{d-1} \mathcal{H}^{d}\right) \otimes \mathcal{R}
$$

with some constant $\lambda_{d-1}$ (the surface area density) and a probability measure $\mathcal{R}$ (the rose of normal directions), a representation, which is in correspondence with (24). Furthermore, $H_{B}(z, t)$ and $\bar{p}(z)$ are independent of $z$, so we write $H_{B}(t)=H_{B}(0, t)$ and $\bar{p}=\bar{p}(0)$. The right sided derivative of $H_{B}(t)$ at $t=0$ is denoted by $H_{B}^{\prime}\left(0_{+}\right)$.

Corollary 5. Let $B \subset \mathbb{R}^{d}$ be a compact set with $o \in B$. Let $Z$ be stationary and a.s. a gentle set such that (23) holds for $D=[0,1]^{d}$. Then

$$
(1-\bar{p}) H_{B}^{\prime}\left(0_{+}\right)=2 \lambda_{d-1} \int_{\mathbb{S}^{d-1}} h(\check{B}, n) \mathcal{R}(d n) .
$$

We return to the general setting, where $Z$ need not be stationary. Let $B$ and $W$ be two non-empty compact subsets of $\mathbb{R}^{d}$ and $o \in W$. Motivated by mathematical morphology, we introduce the hit-or-miss function $G_{(B, W)}(z, \cdot)$ with structuring pair $(B, W)$ of $Z$ at a location $z \in \mathbb{R}^{d}$ by

$$
\begin{aligned}
G_{(B, W)}(z, t) & =P\left(z+t B \subseteq Z, z+t W \subseteq \mathbb{R}^{d} \backslash Z \mid z \notin Z\right) \\
& =P(z \in[Z \ominus t \check{B}] \backslash[Z \oplus t \check{W}] \mid z \notin Z)
\end{aligned}
$$

whenever $\bar{p}(z)<1$. If $\bar{p}(z)=1$, we put $G_{(B, W)}(z, t)=1$. For stationary $Z$, the hitor-miss function does not depend on $z \in \mathbb{R}^{d}$ and we write $G_{(B, W)}(t)=G_{(B, W)}(0, t)$. In the above definition, we did not allow $B=\emptyset$. Formally, however, the first definition in (26) still makes sense and we then have $H_{W}(z, \cdot)=1-G_{(\emptyset, W)}(z, \cdot)$. However, the case $B=\emptyset$ will be excluded in what follows.

Theorem 4. Let $B, W \subset \mathbb{R}^{d}$ be two non-empty compact sets and $o \in W$. Let $Z$ be a.s. a gentle set such that (23) holds for all bounded Borel sets $D \subset \mathbb{R}^{d}$. If $\Lambda_{d-1}\left(\cdot \times \mathbb{S}^{d-1}\right)$ is absolutely continuous with respect to $\mathcal{H}^{d}$ with density $\lambda_{d-1}(\cdot)$, then

$$
\begin{aligned}
& t^{-1}(1-\bar{p}(z)) G_{(B, W)}(z, t) \mathcal{H}^{d}(d z) \\
& \quad \stackrel{v}{\longrightarrow} 2 \lambda_{d-1}(z) \int_{\mathbb{S}^{d-1}}(-h(B \oplus \check{W}, n))^{+} \mathcal{R}(z, d n) \mathcal{H}^{d}(d z),
\end{aligned}
$$

as $t \rightarrow 0_{+}$, where $\stackrel{v}{\longrightarrow}$ denotes the vague convergence of measures.

If, in addition, $Z$ is stationary, then

$$
(1-\bar{p}) G_{(B, W)}^{\prime}\left(0_{+}\right)=2 \lambda_{d-1} \int_{\mathbb{S}^{d-1}}(-h(B \oplus \check{W}, n))^{+} \mathcal{R}(d n) .
$$

Proof. The proof uses ideas from the proofs of Theorem 4.1 and Corollary 4.6 in [6]. Without loss of generality we may assume $B, W \subseteq B(o, 1)$. If $g: \mathbb{R}^{d} \rightarrow \mathbb{R}$ is a continuous function with compact support, we have

$$
\begin{aligned}
\int_{\mathbb{R}^{d}} g(z)(1-\bar{p}(z)) G_{(B, W)}(z, t) \mathcal{H}^{d}(d z) \\
=\mathbb{E}\left[\int_{\mathbb{R}^{d}} g(z) \mathbf{1}_{[Z \ominus t \check{B}] \backslash[Z \oplus t \check{W}]}(z) \mathcal{H}^{d}(d z)\right]
\end{aligned}
$$




$$
\begin{aligned}
= & \mathbb{E}\left[\int_{\mathbb{R}^{d}}\left(g(z)-g\left(\xi_{\partial Z}(z)\right)\right) \mathbf{1}_{[Z \ominus t \check{B}] \backslash[Z \oplus t \check{W}]}(z) \mathcal{H}^{d}(d z)\right] \\
& +\mathbb{E}\left[\int_{\mathbb{R}^{d}} g\left(\xi_{\partial Z}(z)\right) \mathbf{1}_{[Z \ominus t \check{B}] \backslash[Z \oplus t \check{W}]}(z) \mathcal{H}^{d}(d z)\right] \\
= & R_{1}(t)+R_{2}(t) .
\end{aligned}
$$

If $z \in[Z \ominus t \check{B}] \backslash[Z \oplus t \check{W}]$, then $z \in(Z \oplus B(o, t)) \backslash Z$ and thus $\left.\mid z-\xi_{\partial Z}(z)\right) \mid \leq t$. Let $\varepsilon>0$ be an arbitrary real number, denote the support of $g$ by $\operatorname{supp} g$ and define the compact set $D=\operatorname{supp} g \oplus B(o, 1)$. As $g$ is uniformly continuous, there is $0<t_{\varepsilon} \leq 1$ such that $\left|g(z)-g\left(\xi_{\partial Z}(z)\right)\right| \leq \varepsilon$ for all $z \in[Z \ominus t \check{B}] \backslash[Z \oplus t \check{W}]$ whenever $t<t_{\varepsilon}$. For these $t$, we have due to Proposition 4

$$
\begin{aligned}
\left|R_{1}(t)\right| & \leq \varepsilon \mathbb{E} \mathcal{H}^{d}\left(\xi_{\partial Z}^{-1}(D) \cap[Z \ominus t \check{B}] \backslash[Z \oplus t \check{W}]\right) \\
& \leq \varepsilon \sum_{i=1}^{d} i \kappa_{i} \mathbb{E} \int_{N(\partial Z)} \mathbf{1}_{D}(a) \int_{0}^{t} s^{i-1} d s\left|\mu_{d-i}\right|(\partial Z ; d(a, n)) \\
& \leq t \varepsilon \sum_{i=1}^{d} \kappa_{i} t^{i-1} \mathbb{E}\left|\mu_{i}\right|\left(\partial Z ; D \times \mathbb{S}^{d-1}\right) .
\end{aligned}
$$

In view of $\left[9,(2.13)\right.$ and Corollary 2.5] and (23), we obtain $t^{-1}\left|R_{1}(t)\right| \rightarrow 0$, as $t \rightarrow 0_{+}$. According to Corollary 1.(3), the integral in $R_{2}(t)$ satisfies

$$
\begin{aligned}
& t^{-1} \int_{\mathbb{R}^{d}} g\left(\xi_{Z}(z)\right) \mathbf{1}_{[Z \ominus t \check{B}] \backslash[Z \oplus t \check{W}]}(z) \mathcal{H}^{d}(d z) \rightarrow \\
& \quad \rightarrow 2 \int_{N(Z)} g(z)(-h(B \oplus \check{W}, n))^{+} C_{d-1}(Z, d(z, n))
\end{aligned}
$$

as $t \rightarrow 0_{+}$. In view of the dominating terms in the proof of Theorem 1 (which implied Corollary 1.(3)) and (23), Lebesgue's dominated convergence theorem allows to interchange limit and expectation and we get

$$
\begin{aligned}
& \lim _{t \rightarrow 0_{+}} \int_{\mathbb{R}^{d}} g(z) t^{-1}(1-\bar{p}(z)) G_{(B, W)}(z, t) \mathcal{H}^{d}(d z)= \\
& \quad=2 \int_{\mathbb{R}^{d} \times \mathbb{S}^{d-1}} g(z)(-h(B \oplus \check{W}, n))^{+} \Lambda_{d-1}(Z, d(z, n)) .
\end{aligned}
$$

(24) and the fact that $\lambda_{d-1}(\cdot)$ is a density of $\Lambda_{d-1}\left(Z, \cdot \times \mathbb{S}^{d-1}\right)$ completes the proof of the first claim. The stationary case is a direct consequence of this.

\section{Applications to Design Based digital Stereology}

The results for hit distribution functions (and also for hit-or-miss functions) of the previous section can easily be transferred to the design based setting. We focus on gentle sets $A$ which are compact. Although boundedness is not needed, we assume it here to keep notation concise. In the design based approach, the set $A$ under consideration (which was called $Z$ in the last section) is deterministic and the probability $p_{t}$ that a randomly and uniformly translated structuring element $z+t B$ hits $A$, while $z \notin A$, is considered. An asymptotic formula for the first order behavior of $p_{t}$ as $t \rightarrow 0_{+}$is then directly obtained from Theorem 1 and its corollaries. The inexact term of a "random and uniform translation" can easily be made precise by choosing $z$ in a sufficiently large set containing $A \oplus \check{B}$ (when $t \leq 1$ ), which avoids 
edge effects. We will not state these results here but rather show variants which are particularly important in digital stereology.

We introduce the concept of systematic random sampling of sets. Let $x_{1}, \ldots, x_{d}$ be a basis of $\mathbb{R}^{d}$ and let

$$
\mathbb{L}=\left\{n_{1} x_{1}+\ldots+n_{d} x_{d} \mid n_{1}, \ldots, n_{d} \in \mathbb{Z}\right\}
$$

be the lattice generated by this basis. A given lattice $\mathbb{L}$ is generated by infinitely many different bases, but the volume of the parallelepiped $C=\left[o, x_{1}\right] \oplus \ldots \oplus\left[o, x_{d}\right]$ depends only on $\mathbb{L}$ and not on the basis chosen. This number is therefore denoted by $\operatorname{det} \mathbb{L}$. If $\xi$ is a uniform random variable in $C$, the random lattice $\xi+\mathbb{L}$ is a stationary random closed set and we refer to it in what follows as a stationary random lattice, or shorter a stationary lattice. Let $D \subseteq \mathbb{R}^{d}$ be an arbitrary Borel set and let \# $M$ denote the number of elements of a set $M$. We consider different (random) digitizations $\Delta(D)$ of $D . \Delta(\cdot)$ can be seen as a mapping from the Borel sets of $\mathbb{R}^{d}$ to finite subsets of $\xi+\mathbb{L}$. The most common digitization is $\Delta(D)=D \cap(\xi+\mathbb{L})$. It is well known that $(\operatorname{det} \mathbb{L}) \# \Delta(D)$ is an unbiased estimator for the volume of $D$ :

$$
(\operatorname{det} \mathbb{L}) \mathbb{E} \#(D \cap(\xi+\mathbb{L}))=(\operatorname{det} \mathbb{L}) \mathbb{E} \sum_{x \in \xi+\mathbb{L}} \mathbf{1}_{x \in D}=\mathcal{H}^{d}(D)
$$

(a proof uses that $\{x+C: x \in \mathbb{L}\}$ is a tessellation of $\mathbb{R}^{d}$ ). In (28), all points of the randomly translated lattice are counted, which belong to $D$. We therefore talk of systematic random point sampling. The digitization $D \cap(\xi+\mathbb{L})$ is called (random) hit-or-miss digitization. In practice, other digitizations are used, as well. For the pixel digitization, we think of each lattice point $x$ as being the center of small sampling window $x+Q$, where $Q$ is some non-empty compact set, called the sampling element. Often, $Q$ is chosen to be the centered parallelepiped

$$
\left[o, x_{1}\right] \oplus \ldots \oplus\left[o, x_{d}\right]-\left(x_{1}+\ldots+x_{d}\right) / 2
$$

and then $\{x+Q: x \in \mathbb{L}\}$ forms a tessellation of $\mathbb{R}^{d}$. (If $\mathbb{L}=\mathbb{Z}^{d}, d \in\{2,3\}$ and $x_{1}, \ldots, x_{d}$ is the standard basis of $\mathbb{R}^{d}$, the sets $x+[-1 / 2,1 / 2]^{d}, x \in \mathbb{L}$, are called pixels or voxels, respectively). The pixel digitization $D$ consists of all lattice points $x$ for which the corresponding window $x+Q$ hits $D$ and therefore can be represented as $(D \oplus \check{Q}) \cap(\xi+\mathbb{L})$. The hit-or-miss digitization is a special case of the pixel digitization, as $\{o\}$ is a possible choice for $Q$. (The pixel digitization is closely related to the cell-covering digitization of $D$, which is by definition the family of all cells $x+Q$ for which $x \in(D \oplus \check{Q}) \cap(\xi+\mathbb{L})$, if this family is a tessellation of $\mathbb{R}^{d}$, see e.g. [22]). A third digitization in use is the volume-threshold digitization $\left(D \oslash_{\theta} Q\right) \cap(\xi+\mathbb{L})$, already mentioned in the introduction.

We will refine the lattices by scaling them (together with the set $Q$ ) with a factor $t>0$. The resulting digitization is denoted by $\Delta_{t}(\cdot)$. We have $\Delta_{t}(D)=t \Delta(1 / t D)$ for all measurable sets $D$. We first treat volume estimation. If $A$ is a compact gentle set and $\Delta(\cdot)$ is the pixel digitization with compact sampling element $Q \neq \emptyset$, then (28) and Corollary 2.(2) imply

$$
\lim _{t \rightarrow 0_{+}} \frac{t^{d}(\operatorname{det} \mathbb{L}) \mathbb{E} \# \Delta_{t}(A)-\mathcal{H}^{d}(A)}{t}=\int_{\mathbb{S}^{d-1}} h(\check{Q}, n) S_{d-1}(A ; n) .
$$

For the volume-threshold digitization, an analogue result can be stated. 
Proposition 6. Let $A \subset \mathbb{R}^{d}$ be a compact gentle set. Let $\xi+\mathbb{L}$ a stationary lattice and let $\Delta$ be the volume-threshold digitization with compact sampling element $Q \neq \emptyset$ and threshold-parameter $0<\theta \leq 1$. Then

$$
\lim _{t \rightarrow 0_{+}} \frac{t^{d}(\operatorname{det} \mathbb{L}) \mathbb{E} \# \Delta_{t}(A)-\mathcal{H}^{d}(A)}{t}=\int_{\mathbb{S}^{d-1}} f_{\theta}(\check{Q}, n) S_{d-1}(A ; n) .
$$

Proof. From (28), we get

$$
\left.t^{d}(\operatorname{det} \mathbb{L}) \mathbb{E} \# \Delta_{t}(A)=\operatorname{det}(t \mathbb{L})\right) \mathbb{E} \#\left(\left(A \oslash_{\theta} t Q\right) \cap t(\xi+\mathbb{L})\right)=\mathcal{H}^{d}\left(A \oslash_{\theta} t Q\right) .
$$

Corollary 4.(2) implies the assertion.

Proposition 6 was shown in [4] under stronger regularity conditions on $A$ and it was remarked that this result can be used to correct volume estimators of $A$ based on counting the points in $\Delta_{t}(A)$, provided that $t>0$ is small. In the same way, (29) can be used to correct volume estimators for the pixel digitization.

Theorem 5. Let $A$ be a full-dimensional compact gentle set. Let $\xi+\mathbb{L}$ a stationary lattice, $B, W \subset \mathbb{L}$ two non-empty finite subsets of $\mathbb{L}$ and let $\Delta$ either be the pixel digitization or the volume-threshold digitization with compact sampling element $Q \neq$ $\emptyset$ (and threshold-parameter $\theta$ ). Then

$$
N_{t}=\sum_{x \in t(\xi+\mathbb{L})} \mathbf{1}_{\left\{x+t B \subseteq \Delta_{t}(A), x+t W \subseteq t(\xi+\mathbb{L}) \backslash \Delta_{t}(A)\right\}}, \quad t>0,
$$

satisfies

$$
\lim _{t \rightarrow 0_{+}} t^{d-1}(\operatorname{det} \mathbb{L}) \mathbb{E} N_{t}=\int_{\mathbb{S}^{d-1}}(-h(B \oplus \check{W}, n))^{+} S_{d-1}(A ; n) .
$$

Proof. If $\Delta_{t}(A)=(A \oplus t \check{Q}) \cap t(\xi+\mathbb{L})$ is the scaled pixel digitization, then (28) implies

$$
\begin{aligned}
t^{d}(\operatorname{det} \mathbb{L}) \mathbb{E} N_{t} & =\operatorname{det}(t \mathbb{L}) \mathbb{E} \#[(((A \oplus t \check{Q}) \ominus t \check{B}) \backslash((A \oplus t \check{Q}) \oplus t \check{W})) \cap t(\xi+\mathbb{L})] \\
& =\mathcal{H}^{d}([(A \oplus t \check{Q}) \ominus t \check{B}] \backslash[A \oplus t(\check{Q} \oplus \check{W})]) .
\end{aligned}
$$

Replace the variables $C, P, B, Q$ and $W$ in Theorem 1 by $A, \check{Q}, \check{B},\{0\}$ and $\check{Q} \oplus \check{W}$, respectively, to obtain the assertion.

If $\Delta_{t}(A)=\left(A \oslash_{\theta} t \check{Q}\right) \cap t(\xi+\mathbb{L})$ is the scaled volume-threshold digitization, then $(28)$ implies

$$
t^{d}(\operatorname{det} \mathbb{L}) \mathbb{E} N_{t}=\mathcal{H}^{d}\left(\left[\left(A \oslash_{\theta} t \check{Q}\right) \ominus t \check{B}\right] \backslash\left[\left(A \oslash_{\theta} \check{Q}\right) \oplus t \check{W}\right]\right)
$$

and Theorem 2 gives the assertion.

Theorem 5 states that $t^{d-1}(\operatorname{det} \mathbb{L}) N_{t}$ is an asymptotically unbiased estimator for the integral on the right hand side of (30). Note that $N_{t}$ can be calculated alone from the knowledge of the scaled lattice $t(\xi+\mathbb{L})$ and the digitization $\Delta_{t}(A)$. The special case of Corollary 5 , where $\Delta_{t}(A)$ is the hit-or-miss digitization $(Q=\{o\}$ in the pixel digitization) and $A$ is a topologically regular member of the convex ring, has been shown for $d=2$ (in a model based formulation) in [10] and (heuristically) for $d=3$ in [3]. 


\section{REFERENCES}

[1] H. Federer: Curvature measures. Trans. Amer. Math. Soc. 93 (1959), 418-491.

[2] H. Federer: Geometric Measure Theory. Springer, Heidelberg (1969)

[3] P. Gutkowski, E.B.V. Jensen, M. Kiderlen: Directional Analysis of Digitized 3D Images by Configuration Counts. J. Microsc. 216 (2004), 175-185.

[4] P. Hall, I. Molchanov: Corrections for systematic boundary effects in pixel-based area counts. Pattern Recog. 32 (1999), 1519-1528.

[5] D. Hug: Contact distributions of Boolean models. Suppl. Rend. Circ. Mat. Palermo II 65 (2000), 137-181.

[6] D. Hug, G. Last: On support measures in Minkowski spaces and contact distributions in stochastic geometry. Ann. Probab. 28 (2000), 796-850.

[7] D. Hug, G. Last, W. Weil: A survey on contact distributions. Morphology of Condensed Matter. Physics and Geometry of Spatially Complex Systems. Lecture Notes in Physics (K. Mecke, D. Stoyan, eds.), 600 Berlin: Springer (2002), 317-357.

[8] D. Hug, G. Last, W. Weil: Generalized contact distributions of inhomogeneous Boolean models. Adv. Appl. Probab. (SGSA) 34 (2002), 21-47.

[9] D. Hug, G. Last, W. Weil: A local Steiner-type formula for general closed sets and applications. Math. Z. 246 (2004), 237-272.

[10] M. Kiderlen, E.B.V. Jensen: Estimation of the directional measure of planar random sets by digitization. Adv. in Appl. Probab. 35 (2003), 583-602.

[11] G. Matheron: La formule de Steiner pour les érosions. J. Appl. Prob. 15 (1978), 126-135.

[12] I. Molchanov: Statistics of the Boolean model for practitioners and mathematicians, Wiley Series in Probab. and Statist., Wiley, New York (1997).

[13] I. Molchanov: Grey-scale images and random sets. In Mathematical morphology and its applications to image and signal processing, edited by H. Heijmans \& J. Roerdink, Kluwer Acad. Publ., Amsterdam (1998), 247-257.

[14] J. Rataj: Determination of spherical area measures by means of dilation volumes. Math. Nachr. 235 (2002), 143-162.

[15] J. Rataj: On boundaries of unions of sets with positive reach. Beiträge Alg. Geom. (to appear)

[16] J. Rataj, M. Zähle: Curvatures and currents for unions of sets with positive reach, II. Ann. Global Anal. Geom. 20 (2001), 1-21.

[17] R. Schneider: Additive Transformationen konvexer Körper. Geom. Dedicata 3 (1974), 221-228.

[18] R. Schneider, Convex Bodies: The Brunn-Minkowski Theory. Cambridge Univ. Press, Cambridge (1993)

[19] R. Schneider: On the mean normal measures of a particle process. Adv. Appl. Probab. 33 (2001), 25-38.

[20] R. Schneider, W. Weil: Stochastische Geometrie. Teubner, Stuttgart (2000)

[21] D. Stoyan, W.S. Kendall, J. Mecke: Stochastic Geometry and its Applications. 2nd edition, John Wiley, New York (1995)

[22] J. Serra: Image Analysis and Mathematical Morphology, Academic Press, New York (1982)

[23] C. Schütt, E. Werner: The convex floating body. Math. Scand. 66 (1990), 275-290.

[24] M. Zähle: Integral and current representation of Federer's curvature measures. Arch. Math. 46 (1986), 557-567.

[25] M. Zähle: Curvatures and currents for unions of sets with positive reach. Geom. Dedicata 23 (1987), 155-171. 